\title{
Prediction Of Head Degradation Of A Centrifugal Pump Handling Power-Law Fluid
}

\author{
Péter Csizmadia $^{1}$, Dávid Lajos Lukácsi ${ }^{1}$, Sára Till $^{1}$ \\ ${ }^{1}$ Budapest University of Technology and Economics, \\ Faculty of Mechanical Engineering, Department of Hydrodynamic Systems \\ Mủegyetem rkp. 3., Budapest, H-1111, Hungary \\ pcsizmadia@hds.bme.hu; lukacsidavidlajos@edu.bme.hu; still@hds.bme.hu;
}

\begin{abstract}
Operation of centrifugal pumps with non-Newtonian fluids is usually estimated with the help of correction factors. The calculation of these factors is based on methods defined fundamentally for high viscosity Newtonian fluids. A prediction model for head degradation based on the dimensional groups of a centrifugal pump is known for viscous liquids. This model uses experimentally obtained parameters. In this paper, the model's parameters with glycerol solution were validated by comparing the performance curves estimated with the predicted and measured ones. In addition, a possible way of using the estimation model with power-law fluid was investigated. Based on our experimental results at nominal rotational speed, a form for calculating a virtual shear rate was determined, with the help of which the predicted correction factors and head degradation curves at other rotational speeds showed a good agreement with the measurements. The reported results can be considered as a first step in developing an estimation procedure for head degradation of centrifugal pump's operation with power-law fluids.
\end{abstract}

Keywords: centrifugal pump, head degradation, performance curve measurement, non-Newtonian fluid, viscous fluid

\section{Introduction}

The non-Newtonian fluids are widely used in industrial processes and are delivered by pumping between technological processes. For a centrifugal pump, the performance curves are available as datasheets in the catalogue and are given for water. However, if other liquids are supplied with the pump, the performance curves change. Therefore, an accurate estimation of the curves degradation is needed to design the fluid flow system properly.

A considerable amount of literature has been published on the effect of viscosity on the pump operation delivering highly viscous Newtonian fluids [1][2]. Traditionally, these evaluations are based on experimental studies [3], but recently, numerical simulations have also been applied to investigate the pump performance curves for viscous Newtonian fluids [4][6]. Despite a wide range of literature dealing with the topic, just a few proper prediction models are available. These estimation methods provide forms to calculate the head and efficiency degradation compared to the performance with water for highly viscous fluids.

The kinematic viscosity in the existing prediction models is a well-defined parameter for Newtonian fluids but not for the non-Newtonians; it is not constant but depends on the shear rate. Although some research has been carried out on nonNewtonian pump degradation [7]-[10], there is still very little scientific understanding of this problem [11]. Pinelli et al. [12] showed that the shear rate field inside the pump varies widely with power-law fluid. Buratto et al. [13] found that 3D flow structures inside a centrifugal pump obtained with power-law fluid are similar to those obtained with high viscosity Newtonian fluids; therefore, it was proposed to replace it with a virtual Newtonian fluid.

Stepanoff [14] showed that applying the dimensional analysis for a pump gives the describing constants in a dimensionless form, which can be useful to draw conclusions regarding pumping fluids of different viscosities. Gülich [15] formulated the estimation for pumping high viscosity fluids. Based on the earlier works, Ofuchi et al.[16] recently developed a procedure to calculate the degraded points under viscous operation. In their study, a comparison with other models (Hydraulic Institute [1] and KSB [2]) and the limitations of the method were presented as well. Although their approach was validated by measurements, some open questions remained to be answered. However, their practice using normalized dimensionless parameters of the pumps seems to be suitable for alter it to fit the non-Newtonian operation with using virtual Newtonian liquid parameters. 
There are two primary aims of this study: 1 . To validate the Ofuchi model for our pump under viscous operation by comparing the performance curves estimated by the model and the measured ones. 2 . To explore the possible way to extend the model for non-Newtonian operation in the case of a power-law fluid.

\section{Methods}

For validation, measurements were carried out on a centrifugal pump with water and with glycerol solution at different rotations. The correction factors were calculated between the baseline and the measured curves and were compared at three normalized specific speeds with the results of Gülich [15]. The baseline performance curve was modified by the Ofuchi correction procedure [16] to get the predicted degradation of the curves for glycerol solution. Finally, the performance curves from the experiments were compared with the predicted ones.

In an attempt to test the prediction model for non-Newtonian fluids, experiments were performed with a power-law fluid. With choosing an appropriate shear rate to get the average kinematic viscosity of the non-Newtonian fluid, the degraded operation points were also estimated for this fluid.

\subsection{Rheology of the fluids}

As a reference, the head of the centrifugal pump was measured with water at the nominal rotational speed $\left(n_{n}=\right.$ $3355 \mathrm{rpm}$ ). To determine whether the prediction model's parameters were feasible to our pump, we performed measurements with $25 \%$ glycerol solution, a Newtonian fluid but broadly twice as viscous as the water.

The investigated non-Newtonian fluid was a jelly textured bath gel (referred to as "gel"). For determining the rheological parameters, Anton PAAR Physica MCR 301 rotational rheometer was used. The gel showed pseudoplastic rheological behaviour and was estimated by the power-law (Ostwald) rheology model. The rheograms of the fluids and the exact parameters of the describing equations in SI units are given in Fig. 1.

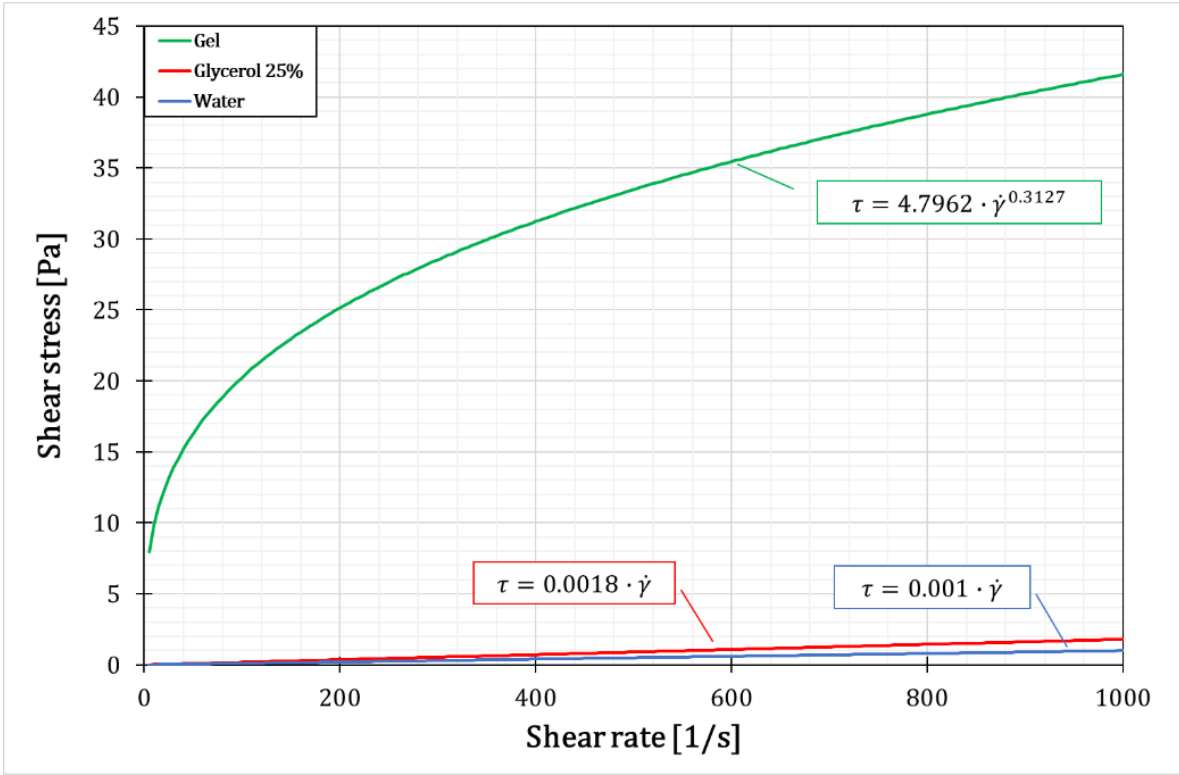

Fig. 1: Rheograms of the test fluids: water, $25 \%$ glycerol solution, gel.

\subsection{Experiments}

For the experiments, a radial centrifugal pump, Wilo-Helix-EXCEL 1602-1/16/E/KS, was built in a test rig. The dimensionless specific speed of the pump was: $n_{q}=21$. The static head $(\mathrm{H},[\mathrm{m}])$ was calculated from the difference between the pressures at the pressure and suction side of the pump, which were measured by calibrated pressure 
transducers. The volume flow rate $\left(\mathrm{Q},\left[\mathrm{m}^{3} / \mathrm{s}\right]\right)$ was determined by a standard orifice meter; the rotational speed (n, [rpm]) was given by the digital data of the pump. The operating points were adjusted using the frequency converter and a gate valve placed at the pressure side of the pump. The baseline characteristic curve of the pump was measured with water. For glycerol solution, five different rotational speeds were set between the minimum $(n 1=1000 \mathrm{rpm})$ and maximum $(n 5=3355 \mathrm{rpm})$ values allowed by the pump control; while in the case of the gel -due to some unstable operation- only two $(n 3=2200 \mathrm{rpm}$ and $n 5=3355 \mathrm{rpm}$ ) were set. Measurements were performed at 8-10 operating points by each rotational speed, taking special care to avoid heating the fluids. During the experiments, the temperature change was allowed a maximum of $1^{\circ} \mathrm{C}$.

\subsection{Prediction method}

To predict the head degradation of the pump in the case of highly viscous or non-Newtonian fluid, the describing parameters had to be normalized [16]. The method required the values at the best efficiency point (referred to with the subscripts of "BEP") for water taken from the pump baseline curve at the nominal rotational speed. From the known dimensionless groups of centrifugal pumps, the following two were relevant for our investigation: 1) rotational Reynolds number $\operatorname{Re}_{\omega}$ and 2) rotational specific speed $\omega_{\mathrm{s}}$ :

$$
\begin{gathered}
R e_{\omega}=\frac{2 \pi n}{60} \frac{D_{2}^{2}}{v} \\
\omega_{s}=\frac{2 \pi n}{60} \frac{Q^{0.5}}{(g H)^{0.75}}
\end{gathered}
$$

Where $\mathrm{n}$ is the actual rotational speed in [rpm], $\mathrm{D}_{2}$ is the impeller outlet diameter in $[\mathrm{m}], \mathrm{Q}$ is the volume flow rate in $\left[\mathrm{m}^{3} / \mathrm{s}\right]$, $\mathrm{H}$ is the head in $[\mathrm{m}], \mathrm{g}$ is the gravitational acceleration in $\left[\mathrm{m} / \mathrm{s}^{2}\right]$, and $v$ is the kinematic viscosity in $\left[\mathrm{m}^{2} / \mathrm{s}\right]$. The normalization removed the geometric parameter from the dimensionless values and gave the normalized Reynolds number $\mathrm{Re}_{\mathrm{n}}$ and normalized specific speed $\omega_{n}$ :

$$
\begin{aligned}
R e_{n} & =\frac{n}{n_{n}} \frac{v_{\text {water }}}{v} \\
\omega_{n} & =\frac{\omega_{s}}{\omega_{s-B E P}}
\end{aligned}
$$

Correction factors of the head $\left(C_{H}\right)$ and volume flow rate $\left(C_{Q}\right)$ were proposed to use in estimations of the pump performance curve for highly viscous fluids. Ofuchi's new method [16] suggested calculating the correction factors based on the normalized dimensionless numbers using the following equations for given normalized specific speeds:

$$
\begin{gathered}
C_{H}=\left.\frac{H}{H_{\text {water }}}\left(\frac{n_{\text {water }}}{n}\right)^{2}\right|_{\omega_{n}} \\
C_{Q}=\left.\frac{Q}{Q_{\text {water }}} \frac{n_{\text {water }}}{n}\right|_{\omega_{n}}
\end{gathered}
$$

However, there is a range of normalized specific speed $0.6 \leq \omega_{n} \leq 1.25$ as a limitation to Eq. (5) and (6), which has to be considered carefully. The simple correlation between the two correction coefficients is known based on Stepanoff [14] and was verified in Ofuchi et al.[16]:

$$
C_{Q}=C_{H}^{1.5}
$$

Eq.(7) assures that only the head correction factor has to be estimated from the baseline pump parameters. The operational specific speed for any operating point (referred with the subscripts of "op") from the baseline water curve is given by:

$$
\omega_{s-o p}=\frac{2 \pi n_{n}}{60} \frac{Q_{o p}{ }^{0.5}}{\left(g H_{o p}\right)^{0.75}}
$$


The modified Reynolds numbers can be calculated for the operating points as well:

$$
R e_{\text {mod }}=\frac{2 \pi n_{o p}}{60} \frac{Q_{B E P}}{v\left(g H_{B E P}\right)^{0.5}} \frac{1}{\omega_{s-o p}}
$$

A correlation between the modified Reynolds number and the correction factor for the head was found and suggested by Gülich [15]:

$$
C_{H}=R e_{\text {mod }}\left(-\frac{6.7}{R e_{\text {mod }}^{0.735}}\right)
$$

With this method, the normalized specific speed with Eq.(2) and Eq.(4) can be calculated for each point of the baseline water curve. In the range of $0.6 \leq \omega_{n} \leq 1.25$ the modified Reynolds number can be determined with Eq.(9) for any rotational speed and any fluid given by the kinematic viscosity value. The $C_{H}$ correction factor for the head is specified by Eq.(10), the one for the volume flow rate $C_{Q}$ with Eq.(7). Finally, the points of the predicted degraded curves are defined with Eq.(11) and Eq.(12):

$$
\begin{aligned}
H_{\text {predicted }} & =C_{H} \cdot H_{o p}\left(\frac{n_{o p}}{n_{n}}\right)^{2} \\
Q_{\text {predicted }} & =C_{Q} \cdot Q_{o p}\left(\frac{n_{o p}}{n_{n}}\right)
\end{aligned}
$$

\section{Results}

\subsection{Viscous operation}

The baseline curve with water and the degraded curves with glycerol solution at five rotational speeds were measured. From the measured performance curves, the actual $C_{H}$ correction factors were calculated at three normalized specific speeds: $\omega_{n}=0.6,1,1.25$. Fig. 2 shows the actual correction factors as the function of the modified Reynolds number defined in Eq. (9) and Eq. (10). The predicted correction factors were in a range of $\pm 5 \%$ around the actual correction factors, except for the values measured at the lowest rotational speed of $n 1=1000 \mathrm{rpm}$. Further investigation is needed to clarify the limitation of the estimation method is in terms of rotational speed. 


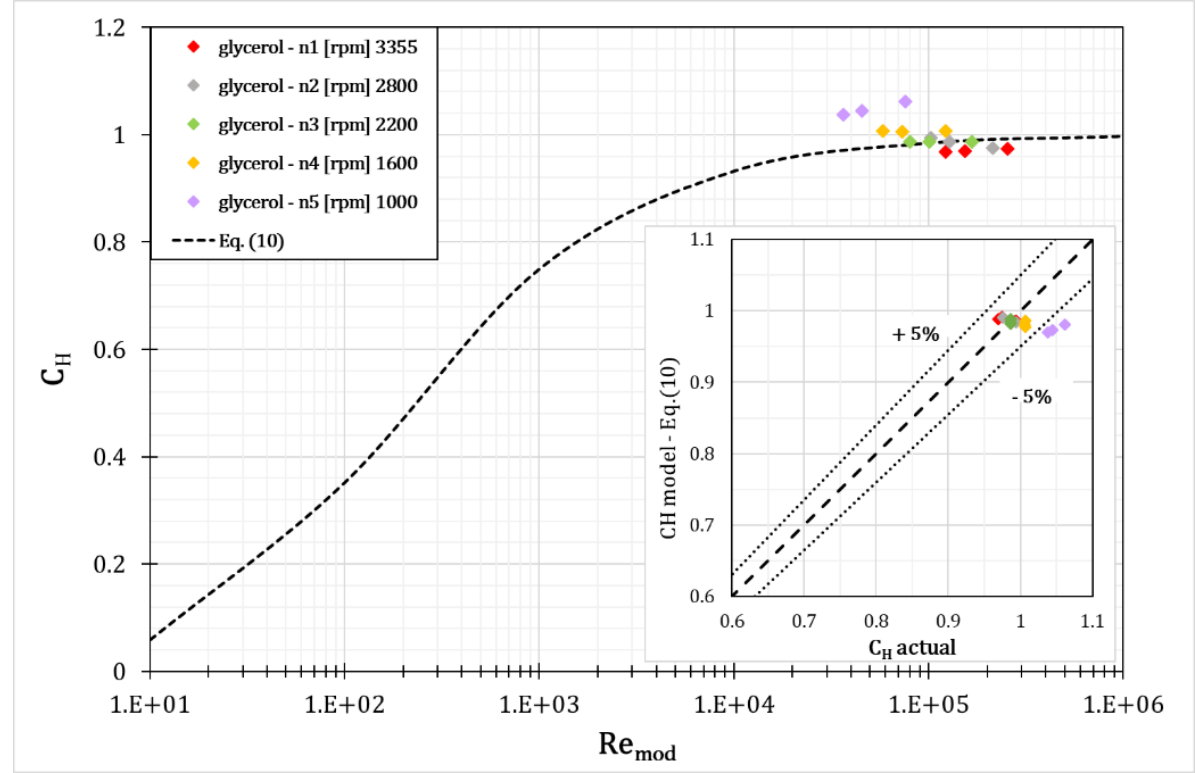

Fig. 2: Actual head correction factors as the function of the modified Reynolds number at three normalized specific speeds for each measured rotational speed with $25 \%$ glycerol solution compared to the Eq. (10)

Fig. 3 shows the measured and predicted performance curves of the pump with glycerol. It can be observed that the degraded head curves at each rotation matched reasonably well.

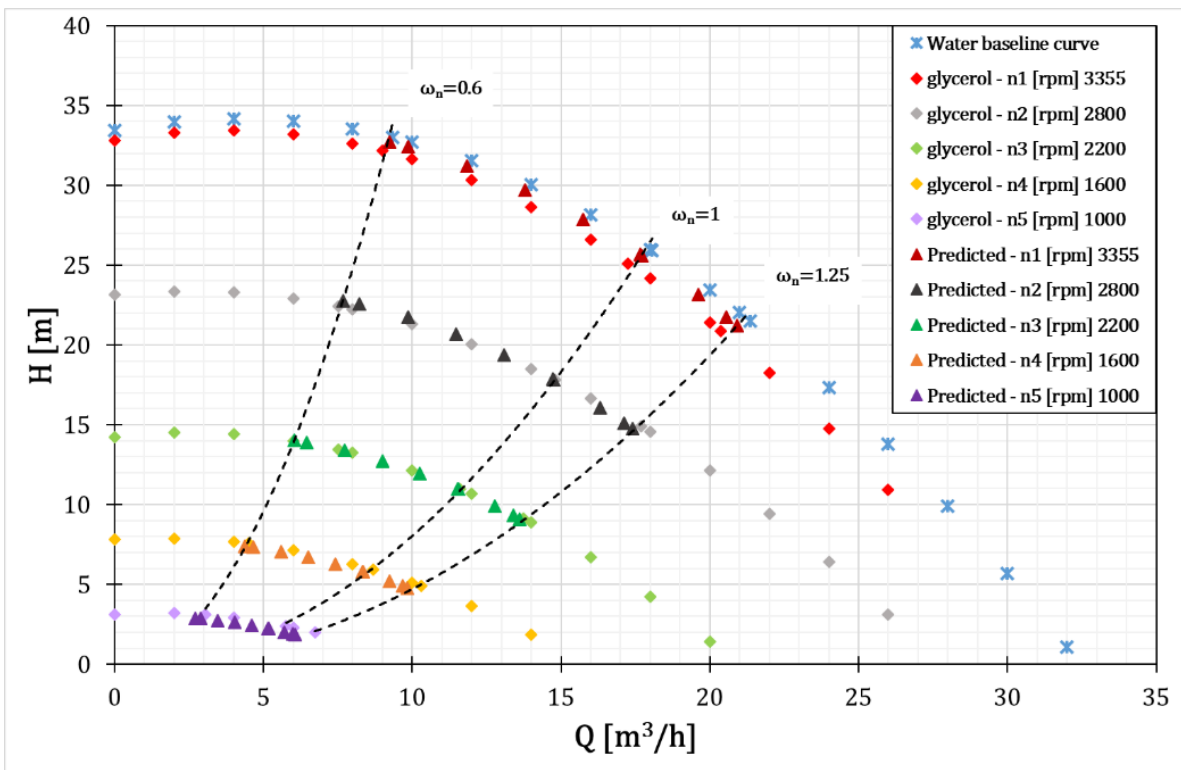

Fig. 3: Measured and predicted performance curves of the pump for each measured rotational speed with $25 \%$ glycerol solution 


\subsection{Non-Newtonian operation}

Two degraded curves have been able to measure with the gel. We assumed an average shear rate could be used by estimating the gel's viscosity, as seen in Buratto et al. [13]. Moreover, we supposed to be a linear correlation between average shear rate $\dot{\gamma}_{a}$ and the actual rotational speed of the pump. The assumption $\dot{\gamma}_{a}[1 / s]=c \cdot n_{o p}[1 / s]$ was completed the baseline rotational speed $n_{n}$ and resulted in constant $c=3.01$ with a coefficient of determination of $R^{2}=0.9971$. average kinematic viscosity was optained from the apparent viscosity at the average shear rate as:

$$
v_{a}=\frac{\mu_{a}}{\rho}=\frac{4.7962 \cdot \dot{\gamma}_{a}^{(0.3127-1)}}{\rho}
$$

Where $\mu_{a}$ is the average apparent viscosity in $[\mathrm{Pa} \cdot \mathrm{s}]$, and $\rho$ is the density in $\left[\mathrm{kg} / \mathrm{m}^{3}\right]$. With this substitution of the kinematic viscosity with one average - virtual- value at power-law fluid, the prediction model steps and parameters were the same as the viscous operation. The measured and predicted performance curves with gel are shown in Fig. 4. It can be seen that this estimation resulted quite well at the lowest rotation speed.

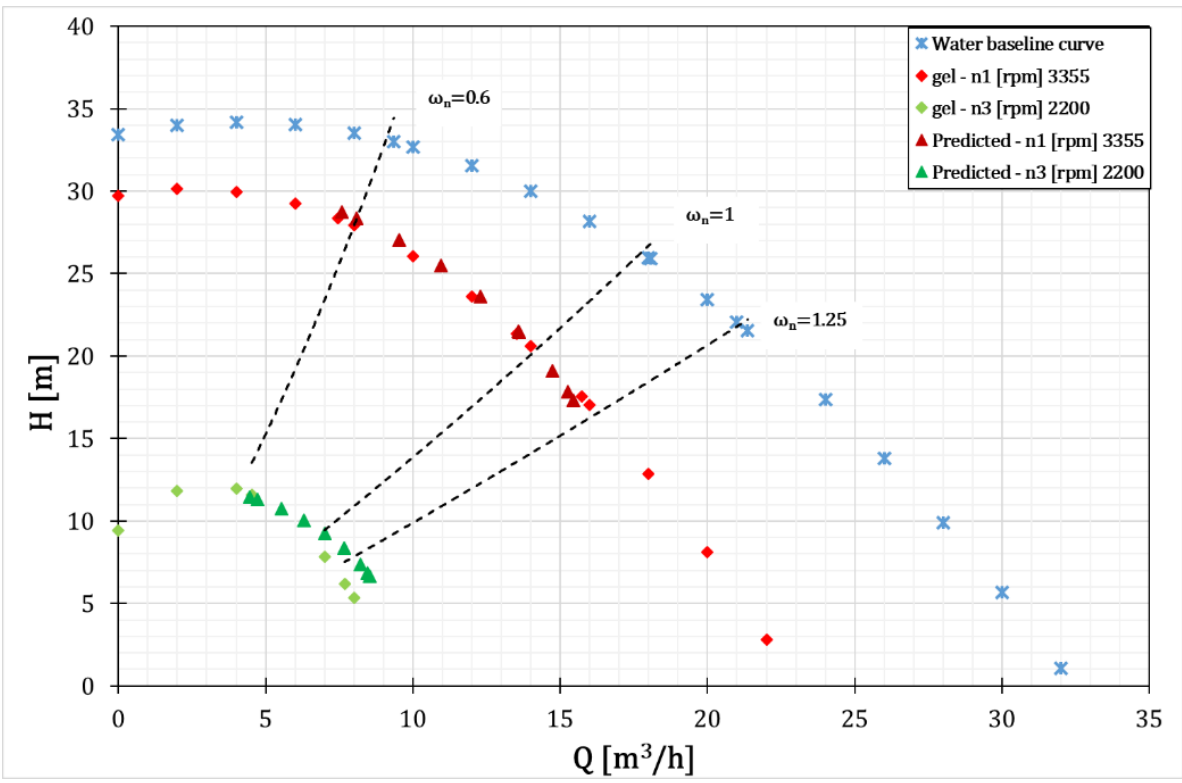

Fig. 4: Measured and predicted performance curves of the pump for the two measured rotational speeds with the gel

\section{Conclusions}

The following conclusions can be drawn from the present study, which shows the results of experiments carried out with a centrifugal pump with the dimensionless specific speed of $n_{q}=21$. The measurements with viscous $25 \%$ glycerol solution at five different speeds fit well with the predicted data with Ofuchi's model. An average-virtual- shear rate was introduced to determine the apparent viscosity of the power-law fluid and thereby to describe the degradation caused by the non-Newtonian gel. With the tested fluids, the pump's performance curves could be estimated with the established methods at different rotational speeds. We want to extend the presented method using other fluids and measuring different pumps as a further step. 


\section{Acknowledgements}

The work was supported by the New National Excellence Program No. ÚNKP-21-5-BME-381 and ÚNKP-20-5-BME156 of the Ministry of Innovation and Technology Hungary, awarded by Péter Csizmadia and by the János Bolyai Research Scholarship of Hungary.

\section{References}

[1] G. R. Addie, A. S. Roudnev, and A. Sellgren, "The new ANSI/HI centrifugal slurry pump standard," J. South. African Inst. Min. Metall., vol. 107, no. 6, pp. 403-409, 2007, [Online]. Available: https://www.saimm.co.za/Journal/v107n06p403.pdf.

[2] KSB, Selecting Centrifugal Pumps, 4th ed. Frankenthal, Germany: KSB Aktiengeselschaft, Zentrale Kommunikation, 2005.

[3] J. F. Gülich, "Pumping highly viscous fluids with centrifugal pumps - Part 2," World Pumps, vol. 1999, no. 395, pp. 30-34, 1999, DOI: 10.1016/S0262-1762(00)87528-8.

[4] R. Torabi and S. A. Nourbakhsh, "The effect of viscosity on performance of a low specific speed centrifugal pump," Int. J. Rotating Mach., vol. 2016, 2016, DOI: 10.1155/2016/3878357.

[5] H. Yousefi, Y. Noorollahi, M. Tahani, R. Fahimi, and S. Saremian, "Numerical simulation for obtaining optimal impeller's blade parameters of a centrifugal pump for high-viscosity fluid pumping," Sustain. Energy Technol. Assessments, vol. 34, no. February, pp. 16-26, 2019, DOI: 10.1016/j.seta.2019.04.011.

[6] E Ofuchi E. M., Stel H., Sirino T., Vieira T., Ponce F., Chiva S., Morales R., "Numerical investigation of the effect of viscosity in a multistage electric submersible pump," Eng. Appl. Comput. Fluid Mech., vol. 11, no. 1, pp. 258-272, 2017, DOI: 10.1080/19942060.2017.1279079.

[7] J. J. N. Kalombo, R. Haldenwang, R. P. Chhabra, and V. G. Fester, "Centrifugal pump derating for non-newtonian slurries," J. Fluids Eng. Trans. ASME, vol. 136, no. 3, pp. 1-11, 2014, DOI: 10.1115/1.4025989.

[8] C. I. Walker and A. Goulas, "Performance Characteristics of Centrifugal Pumps When Handling Non-Newtonian Homogeneous Slurries," Proc Instn Mech Engrs Vol, vol. 198A, no. 1, pp. 41-48, 1984, DOI: 10.1243/PIME_PROC_1984_198_006_02.

[9] L. J. W. Graham, L. Pullum, P. Slatter, G. Sery, and M. Rudman, "Centrifugal pump performance calculation for homogeneous suspensions," Can. J. Chem. Eng., vol. 87, no. 4, pp. 526-533, 2009, DOI: 10.1002/cjce.20192.

[10] N. Heywood and P. Slatter, "Deration of Centrifugal Pumps for Non-Newtonian Slurries," in 19th International Conference on Transport and Sedimentation of Solid Particles, 2019, no. September, pp. 219-227.

[11] Buratto C., Occari M., Aldi N., Casari N., Pinelli M., Spina P. R., Suman A., "Centrifugal pumps performance estimation with non-Newtonian fluids: Review and critical analysis," 12th Eur. Conf. Turbomach. Fluid Dyn. Thermodyn. ETC 2017, no. January, 2017, DOI: 10.29008/etc2017-248.

[12] Aldi N., Buratto C., Casari N, Dainese D., Mazzanti V., Mollica F., Munari E., Occari M., Pinelli M., Randi S., Spina P. R., Suman A., "Experimental and Numerical Analysis of a Non-Newtonian Fluids Processing Pump," Energy Procedia, vol. 126, pp. 762-769, 2017, DOI: 10.1016/j.egypro.2017.08.247.

[13] C. Buratto, M. Pinelli, P. R. Spina, A. Vaccari, and C. Verga, "CFD study on special duty centrifugal pumps operating with viscous and non-Newtonian fluids," 11th Eur. Conf. Turbomach. Fluid Dyn. Thermodyn. ETC 2015, no. i, pp. 1$13,2015$.

[14] A. J. Stepanoff, Centrifugal and axial flow pumps, 2nd ed. Malabar, Florida: Krieger Publishing Company, 1957.

[15] J. F. Gülich, Centrifugal pumps, 2nd ed. Springer Berlin Heidelberg, 2010.

[16] E. M. Ofuchi, J. M. C. Cubas, H. Stel, R. Dunaiski, T. S. Vieira, and R. E. M. Morales, "A new model to predict the head degradation of centrifugal pumps handling highly viscous flows," J. Pet. Sci. Eng., vol. 187, no. November 2019, p. 106737, 2020, DOI: 10.1016/j.petrol.2019.106737. 